\title{
On the strength of space-time singularities
}

\author{
János Kánnár and István Rácz \\ Central Research Institute for Physics, H-1525 Budapest 114, P.O.B. 49, Hungary
}

(Received 27 June 1991; accepted for publication 2 April 1992)

New integral conditions are proposed that are sufficient for the existence of conjugate

pointpairs along causal geodesics. As to maximal incomplete causal geodesics the upper bound on the rate of possible divergency of the tidal curvature is examined.

\section{INTRODUCTION}

Inextendible causal geodesics of finite total affine length have been proved to be inevitably present in the general relativistic models of universe and gravitational collapse by the singularity theorems of Penrose, Hawking, and Geroch. ${ }^{1-3}$ These are existence theorems and they tell almost nothing about the nature of the spacetime singularities. Incompleteness of causal geodesics does not imply that some of the "physical" quantities blow up along them. On the other hand, as shown first by Tipler, ${ }^{4}$ one can give an upper bound on the rate of the possible divergency of the Ricci part of the tidal curvature along incomplete maximal causal geodesics. Here, maximality of a causal curve means that it is the longest one in its close neighborhood in the space of causal curves. The maximality condition seems to be too strong a restriction; nevertheless, the presence of causal geodesics having this property turned to be a generic feature of singular space-times. Namely, as shown by Szabados, ${ }^{5}$ the conditions imposed on space-times in singularity theorems automatically imply, beyond the existence of incomplete causal geodesics, their maximality.

In this paper, we are going to generalize the upper bound results of Tipler ${ }^{4,6}$ Newman, ${ }^{7}$ and Szabados. ${ }^{8,9}$ In particular, we examine not only the Ricci part or the conformal electric curvature, but also the separate components of the tidal force tensor, and consider, besides the null ones, the timelike geodesics as well. In the argument, some aspects of the Morse index theory will be incorporated instead of the conventional approach relying on the examination of the solutions of the Raychaudhuri equation. Not only more powerful theorems can be developed by this approach, but also the null and the timelike cases can be treated on equal footing.

In the next section some notions and results are recalled, in connection with the Morse index theory. In Sec. III some sufficient conditions are derived, assuring the existence of conjugate pointpairs along causal geodesics in terms of an integral of a certain component of the tidal force tensor. In Sec. IV, these results are applied to give restrictions on the above-mentioned integrals along incomplete maximal causal geodesics. Finally, some concluding remarks are given.
Our conventions and notations are the same as those of Ref. 2, so, e.g., the space-time metric $g$ is considered to be a smooth Lorentz metric of signature +2 and for the curvature tensor $R$ we have that $R(X, Y) Z=\nabla_{X}\left(\nabla_{Y}\right) Z$ $-\nabla_{Y}\left(\nabla_{X}\right) Z-\nabla_{[X, Y]} Z$, where $X, Y, Z$ are arbitrary vector fields and $\nabla$ denotes the torsion-free covariant derivative operator determined by the metric.

\section{PRELIMINARIES}

In this section some well-known definitions and results will be recalled that we shall need.

A causal curve $\gamma:[a, b] \rightarrow M$ from $\gamma(a)$ to $\gamma(b)$ is said to be maximal if among all causal curves from $\gamma(a)$ to $\gamma(b)$ generated by (infinitesimal) continuous piecewise$C^{\mathrm{l}}$ variations of $\gamma$ it is the longest one. A future inextendible causal curve $\gamma:[a, b) \rightarrow M$ is said to be maximal if for any $b^{\prime} \in(a, b)$ the segment $\gamma:\left[a, b^{\prime}\right] \rightarrow M$ is maximal. As detailed examinations show (see Refs. 1 and 10) $\gamma:[a, b] \rightarrow M$ is maximal if and only if it is geodesic and there is no conjugate pointpair between $\gamma(a)$ and $\gamma(b)$ along $\gamma$. Necessary and sufficient conditions for the existence of conjugate pointpairs along causal geodesics can be given in terms of the definiteness of the (Morse) index form (see Ref. 10). For the present purposes, however, use of the so-called timelike and null index lemmas, given by Harris, ${ }^{11}$ seems to be more economical, so we shall recall them.

Let $\gamma:[a, b] \rightarrow M$ be a causal geodesic in $M$ with an affine parameter $t$ (which is the proper time if $\gamma$ is timelike), and denote by $V(\gamma)$ the real vector space of all continuous piecewise- $C^{1}$ vector fields along $\gamma$. The index form $I: V(\gamma) \times V(\gamma) \rightarrow \mathbb{R}$ is the symmetric bilinear form given by the integral

$$
I(X, Y):=\int_{a}^{b}\left[g\left(\nabla_{T} X, \nabla_{T} Y\right)-g(R(X, T) T, Y)\right] d t,
$$

for any $(X, Y) \in V(\gamma) \times V(\gamma)$, where $T$ denotes the tangent vector of $\gamma$. Then the "first or timelike index lemma" for timelike geodesics is given as follows: ${ }^{11}$

Timelike Index Lemma: Let $\gamma:[a, b] \rightarrow M$ be a timelike geodesic with no points conjugate to $\gamma(a)$ and $X$ be a continuous piecewise- $C^{\mathrm{l}}$ vector field along $\gamma$, perpendicular to $\gamma$, with $X(a)=0$. Let, furthermore, $J$ be a perpen- 
dicular Jacobi field along $\gamma$ with $J(a)=0$ and $J(b)$ $=X(b)$. Then $I(X, X)>I(J, J)$, and $I(X, X)=I(J, J)$ only if $X=J$.

If $\gamma:[a, b] \rightarrow M$ happens to be a null geodesic, the situation is different, because the tangent vector $T$ of $\gamma$ belongs to its own perpendicular space. However, a slightly modified version of the above lemma can be prove, even in this case. ${ }^{11}$

Null Index Lemma: Let $\gamma:[a, b] \rightarrow M$ be a null geodesic with no points conjugate to $\gamma(a)$ and $X$ be a continuous piecewise- $C^{1}$ vector field along $\gamma$, perpendicular to $\gamma$, with $X(a)=0$. Let, furthermore, $J$ be a perpendicular Jacobi field along $\gamma$ with $J(a)=0$ and $J(b)=X(b)$. Then $I(X, X)>I(J, J)$, and $I(X, X)=I(J, J)$, only if $X-J$ is everywhere parallel to $T$.

\section{THE EXISTENCE OF CONJUGATE POINTS}

By the application of the above index lemmas, some conditions will be presented that are sufficient for the existence of conjugate points along causal geodesics.

Throughout the following two sections, for a given causal geodesic $\gamma E$ will denote one of the unit spacelike vector fields parallelly propagated along and perpendicular to $\gamma$. Let $\gamma:[a, b] \rightarrow M$ be a causal geodesic and $E$ be a vector field along $\gamma$. Let, furthermore, $f:[a, b] \rightarrow \mathbb{R}$ be a continuous piecewise- $C^{2}$ function, and $X$ be the vector field $X:=f E$ along $\gamma$. Clearly, $X$ belongs to $V(\gamma)$ and the index form takes the value

$$
I(X, X)=\int_{a}^{b}\left[\left(f^{\prime}(t)\right)^{2}-(f(t))^{2} k_{E \wedge T}(t)\right] d t,
$$

on the pair $(X, X) \in V(\gamma) \times V(\gamma)$, where the prime denotes differentiation with respect to $t$, and the function $k_{E \wedge T}(t)$ is defined as

$$
k_{E \wedge T}(t):= \begin{cases}-K(\gamma(t), \sigma), & \text { if } \gamma \text { is timelike, } \\ K_{T}(\gamma(t), \sigma), & \text { if } \gamma \text { is null, }\end{cases}
$$

where $K(\gamma(t), \sigma)$ [resp., $K_{T}(\gamma(t), \sigma)$ ] denotes the timelike (resp., null) sectional curvature (in the null case with respect to $T$ ) of the two-dimensional timelike (resp., null) linear subspace $\sigma$ in $T_{\gamma(t)} M$ generated by the basis $\{E, T\}{ }^{10,11}$ Recall that $K(\gamma(t), \sigma)$ [resp., $\left.K_{T}(\gamma(t), \sigma)\right]$ may be calculated by choosing a basis $\{X, Y\}$ (resp., $\{X, T\}$ ) for $\sigma$ and setting

$$
\begin{aligned}
& K(\gamma(t), \sigma):=-\frac{g(R(X, Y) X, Y)}{g(X, X) g(Y, Y)-[g(X, Y)]^{2}}, \\
& \left(\text { resp., } K_{T}(\gamma(t), \sigma):=-\frac{g(R(X, T) X, T)}{g(X, X)}\right) .
\end{aligned}
$$

Hereafter the function $k_{E \wedge T}(t)$, the value of which depends only on the subspace $\sigma$ and not on the basis chosen, will be referred to as the curvature function. It is worth noting that $k_{E \wedge T}(t)$ is equal to the tidal force component $R_{1414}(t)$ of the curvature tensor in such a parallelly propagated orthonormal (resp., pseudo-orthonormal) basis $\left\{E_{a}\right\}$ that $E_{1}=E$ and $E_{4}=T$.

In what follows we shall apply a selected class of functions, denoted by $\mathscr{F}[a, b]$, to get restrictions on the timelike (resp., null) sectional curvature. Let $\delta>0$ and $g:[0, \delta] \rightarrow \mathbb{R}$ be such a $C^{2}$ function that $-1 \leqslant g \leqslant 1, g(0)=0$ and $g(\delta)=1$. The set of functions having these properties will be denoted by $G_{\delta}$. Now, then let the function $f_{\delta}:[a, b] \rightarrow \mathbb{R}$ be defined as follows:

$$
f_{\delta}(t):= \begin{cases}g^{\circ}(t-a), & \text { if } a \leqslant t \leqslant a+\delta, \\ 1, & \text { if } a+\delta \leqslant t \leqslant b-\delta, \\ h \circ(b-t), & \text { if } b-\delta \leqslant t \leqslant b,\end{cases}
$$

for some $g, h \in G_{\delta}$, where $0<\delta \leqslant \frac{1}{2}(b-a)$. The set of functions $f_{\delta}$ defined in this way will be denoted by $\mathscr{F}[a, b]$. Clearly, the members of $\mathscr{F}[a, b]$ are continuous (at least) piecewise- $C^{2}$ functions.

Before presenting our main result we shall prove two lemmas.

Lemma 3.1: Let $t_{1}, t_{2} \in \mathbb{R}$ and $0<\delta<\left(t_{2}-t_{1}\right) / 2$. Furthermore, let $k(t): \mathbb{R} \rightarrow \mathbb{R}$ be a continuous function that does not change sign in each of the intervals $\left[t_{1}-\delta, t_{1}+\delta\right]$ and $\left[t_{2}-\delta, t_{2}+\delta\right]$. Then, for any given $g, h \in G_{\delta}$, there exist such an $a \in\left[t_{1}-\delta, t_{1}\right]$ and a $b \in\left[t_{2}, t_{2}+\delta\right]$ that

$$
\int_{a}^{b}\left(f_{\delta}(t)\right)^{2} k(t) d t=\int_{t_{1}}^{t_{2}} k(t) d t
$$

is satisfied, where $f_{\delta}$ is defined through $g$ and $h$.

Proof: It is clear that (3.2) will be satisfied if for any given $g, h \in G_{\delta}$ there exist such an $a$ and a $b$, for which

$$
\int_{a}^{a+\delta}\left(f_{\delta}(t)\right)^{2} k(t) d t=\int_{t_{1}}^{a+\delta} k(t) d t
$$

and

$$
\int_{b-\delta}^{b}\left(f_{\delta}(t)\right)^{2} k(t) d t=\int_{b-\delta}^{t_{2}} k(t) d t
$$

We restrict the proof to the case of $a$, the existence of a proper $b$ in (3.4) can be shown in an analogous way. Let $g \in G_{\delta}$ and let the function $\Gamma:\left[t_{1}-\delta, t_{1}\right] \rightarrow \mathbb{R}$ be defined as

$$
\begin{aligned}
\Gamma(\alpha):= & \int_{\alpha}^{t_{1}}\left[g^{\circ}(t-\alpha)\right]^{2} k(t) d t \\
& -\int_{t_{1}}^{\alpha+\delta}\left(1-\left[g^{\circ}(t-\alpha)\right]^{2}\right) k(t) d t .
\end{aligned}
$$


Then (3.3) must have a solution $a \in\left[t_{1}-\delta, t_{1}\right]$ if the function $\Gamma$ vanishes somewhere in the interval $\left[t_{1}-\delta, t_{1}\right]$. Since $k(t)$ does not change sign on $\left[t_{1}-\delta, t_{1}\right]$, one of the alternatives, (a) $k(t) \geqslant 0$ on $\left[t_{1}-\delta, t_{1}\right]$ [whence $\Gamma\left(t_{1}-\delta\right) \geqslant 0$ and $\Gamma\left(t_{1}\right) \leqslant 0$ ]; and (b) $k(t) \leqslant 0$ on $\left[t_{1}-\delta, t_{1}\right]$ [whence $\Gamma\left(t_{1}\right.$ $-\delta) \leqslant 0$ and $\left.\Gamma\left(t_{1}\right) \geqslant 0\right)$; holds.

As the function $\Gamma$ is continuous in the interval $\left[t_{1}\right.$ $\left.-\delta, t_{1}\right]$ it must vanish in both cases somewhere in $\left[t_{1}\right.$ $\left.-\delta, t_{1}\right]$.

Lemma 3.2: Let $\delta>0$ and $g \in G_{\delta}$. Then we have

$$
\tilde{I}(g):=\int_{0}^{\delta}\left(g^{\prime}(t)\right)^{2} d t \geqslant \frac{1}{\delta},
$$

and $\tilde{I}(g)=\frac{1}{\delta}$ only if $g(t)=t / \delta$.

Proof: Let $\delta>0$ and consider a smooth oneparameter family $g_{\lambda}$ of functions in $G_{\delta}$. Then the function $g_{\lambda=Q}$ extremizes the functional $\tilde{I}$ whenever $\delta \widetilde{I}$ : $=d I /\left.d \lambda\right|_{\lambda=0}=0$. Now, for the first variation $\delta \widetilde{I}$ of the functional $I$, we get that

$$
\delta \widetilde{I}=2 \int_{0}^{\delta} g^{\prime}(t) \delta g^{\prime}(t) d t
$$

where $\delta g:=d g_{\lambda} /\left.d \lambda\right|_{\lambda=0}$. Clearly, $\delta^{n} g(a \rightarrow 0)=\delta^{n} g(b \rightarrow \delta)$ $=0$ for any $n \in\{1,2, \ldots\}$. Upon integrating (3.6) by parts, we get that

$$
\delta \tilde{I}=-2 \int_{0}^{\delta} g^{\prime \prime}(t) \delta g(t) d t
$$

i.e., $g_{\lambda=0} \in G_{\delta}$ extremizes $\widetilde{I}$, only if it is just the function $g(t)=t / \delta$. To see that $\widetilde{I}$ takes the minimum value at $g(t)=t / \delta$, it is enough to consider the second variation,

$$
\delta^{2} \widetilde{I}=\int_{0}^{\delta} 2\left(\delta g^{\prime}(t)\right)^{2} d t
$$

which is greater than zero for any nontrivial variation of $g(t)=t / \delta$.

Now, we can prove our main result, which we shall apply in the remaining part of this paper.

Proposition 3.3: Let $\gamma:[a, b] \rightarrow M$ be a causal geodesic in $M$, and $t_{1}, t_{2} \in(a, b)$ and $0<\delta \leqslant\left(t_{2}-t_{1}\right) / 2$, so that $\left[t_{1}\right.$ $\left.-\delta, t_{2}+\delta\right] \subset[a, b]$. Suppose that there exists a vector field $E$ along $\gamma$ so that the curvature function $k_{E \wedge T}(t)$ does not change sign in each of the intervals $\left[t_{1}-\delta, t_{1}+\delta\right]$ and $\left[t_{2}-\delta, t_{2}+\delta\right]$. Furthermore, suppose that

$$
\int_{t_{1}}^{t_{2}} k_{E \wedge T}(t) d t \geqslant \frac{2}{\delta}
$$

is satisfied. Then there exists a conjugate pointpair along $\gamma$ on the segment between $\gamma\left(t_{1}-\delta\right)$ and $\delta\left(t_{2}+\delta\right)$.

Proof: Suppose, on the contrary, that there is no conjugate pointpair along $\gamma$ on the segment between $\gamma\left(t_{1}-\delta\right)$ and $\gamma\left(t_{2}+\delta\right)$. According to Lemma 3.1., to the interval $\left[t_{1}, t_{2}\right]$, and for any given $g, h \in G_{\delta}$, there exist such an $\widetilde{a} \in\left[t_{1}\right.$ $\left.-\delta, t_{1}\right]$ and a $\widetilde{b} \in\left[t_{2}, t_{2}+\delta\right]$ that

$$
\int_{t_{1}}^{t_{2}} k_{E \wedge T}(t) d t=\int_{\tilde{a}}^{\tilde{b}}\left(f_{\delta}(t)\right)^{2} k_{E \wedge T}(t) d t
$$

is satisfied, where $f_{\delta}$ is given through the functions $g$ and $h$. Hence, the index form takes the value

$$
I(X, X)=\int_{\tilde{a}}^{\tilde{b}}\left(f_{\delta}^{\prime}(t)\right)^{2} d t-\int_{t_{1}}^{t_{2}} k_{E \wedge T}(t) d t
$$

for $(X, X) \in V(\gamma) \times V(\gamma)$, where $X=f_{\delta} E$. Let $g(t)=h(t)$ : $=t / \delta$. Then we have

$$
\int_{\tilde{a}}^{\widetilde{b}}\left(f_{0}^{\prime}(t)\right)^{2} d t=\frac{2}{\delta}
$$

Furthermore, since

$$
\int_{t_{1}}^{t_{2}} k_{E \wedge T}(t) d t \geqslant \frac{2}{\delta}
$$

we get that $I(X, X) \leqslant 0$ is satisfied. However, according to the timelike (resp., null) index lemma, this implies that $X$ vanishes along $\gamma$ (or in the null case it is purely tangential) if $\gamma$ has no conjugate point to $\gamma(\widetilde{a})$ on the segment between $\gamma(\widetilde{a})$ and $\gamma(\widetilde{b})$. Since neither is true, the inverse assumption must fail, i.e., $\gamma$ contains a conjugate pointpair between $\gamma\left(t_{1}-\delta\right)$ and $\gamma\left(t_{2}+\delta\right)$.

In view of Lemma 3.2. One can say that the lower bound $2 / \delta$ appearing in expression (3.7) cannot be improved in the set of functions $\mathscr{F}[a, b]$.

As a direct consequence of the above proposition the next statement can be proved.

Corollary 3.4: Let $\gamma:(-\infty,+\infty) \rightarrow M$ be a complete causal geodesic in $M$. Suppose that there exists a vector field $E$ along $\gamma$ so that the curvature function $k_{E \wedge T}(t)$ is non-negative along $\gamma$, while being positive somewhere. Then there exists a conjugate pointpair along $\gamma$.

Proof: The above condition on the curvature function $k_{E \wedge T}(t)$ implies that

$$
\int_{-\infty}^{+\infty} k_{E \wedge T}(t) d t>0
$$

and so there must be such parameter values $t_{1}, t_{2} \in \mathbb{R}$ and a positive number $\delta$ to $t_{1}$ and $t_{2}$ that $0<\delta \leqslant\left(t_{1}-t_{2}\right) / 2$ and $\int_{t_{1}}^{t_{2}} k_{E \wedge T}(t) d t>2 / \delta$ are satisfied. Then, according to Proposition 3.3, there exists a conjugate pointpair along $\gamma$ between $\gamma\left(t_{1}-\delta\right)$ and $\gamma\left(t_{2}+\delta\right)$.

Clearly, the restriction on the curvature function $k_{E \wedge} r^{(t)}$ involved in the previous result plays the same role as the timelike (resp., null) convergence and the 
so-called genericness conditions, together in the singularity theorems of Hawking and Penrose. ${ }^{1,2}$ However, it is worth noting that there can exist such a situation in which our condition is satisfied while the timelike (resp. null) convergence condition is violated everywhere along some causal geodesics. Consequently, a generalization of the Hawking-Penrose singularity theorem can be given.

It turned out that the timelike (resp., null) convergence condition can be further weakened. Details and results on this line of examinations can be found in Ref. 12.

\section{THE BEHAVIOR OF THE TIDAL FORCE TENSOR}

In this section the behavior of the tidal force tensor along maximal incomplete causal geodesics will be examined. First the diagonal elements then the off-diagonal ones will be considered.

\section{A. The diagonal elements}

In the remaining part of this paper we shall apply the following simplified version of Proposition 3.3.

Proposition 4.1.1: Let $\gamma:[a, b] \rightarrow M$ be a maximal causal geodesic segment in $M$. Then for all $t_{1}, t_{2} \in(a, b)$, satisfying the relation $\left[\left(3 t_{1}-t_{2}\right) / 2,\left(3 t_{2}-t_{1}\right) / 2\right] \subset[a, b]$, we have the inequality

$$
\int_{\ell_{1}}^{t_{2}} k_{E \wedge T}(t) d t<4 / t_{2}-t_{1}
$$

for any curvature function $k_{E \wedge T}(t)$ that does not change sign on the interval $\left[\left(3 t_{1}-t_{2}\right) / 2,\left(3 t_{2}-t_{1}\right) / 2\right]$.

Proof: Clearly, whenever $k_{E \wedge T}(t) \leqslant 0$ on $\left[\left(3 t_{1}-t_{2}\right)\right.$ / $\left.2,\left(3 t_{2}-t_{1}\right) / 2\right]$, inequality (4.1) will be satisfied. Suppose, on the contrary, that there are $t_{1}, t_{2} \in(a, b)$ so that

$$
\int_{t_{1}}^{t_{2}} k_{E \wedge T}(t) d t \geqslant \frac{4}{t_{2}-t_{1}}
$$

and $k_{E \wedge T}(t) \geq 0$ on $\left[\left(3 t_{1}-t_{2}\right) / 2,\left(3 t_{2}-t_{1}\right) / 2\right]$. In this case, with the choice $\delta:=\left(t_{2}-t_{1}\right) / 2$, the conditions of Propositions 3.3 are satisfied. Consequently, there exists a conjugate pointpair along $\gamma$ between $\gamma\left(\left(3 t_{1}-t_{2}\right) / 2\right)$ and $\gamma\left(\left(3 t_{2}-t_{1}\right) / 2\right)$, so the geodesic $\gamma$ cannot be maximal.

Proposition 4.1.2: Let $\gamma:\left(-\infty, t_{0}\right) \rightarrow M$ be a future incomplete casual geodesic. Suppose that there exists an interval $\left[t^{*}, t_{0}\right)$ and a vector field $E$ along $\gamma$ so that the curvature function $k_{E \wedge T}(t)$ does not change sign on $\left[t^{*}, t_{0}\right)$ and

$$
\lim _{t \rightarrow t_{0}} \inf _{\left(t, t_{0}\right)}\left\{\left(t-t_{0}\right)^{2} k_{E \wedge T}(t)\right\}>9 .
$$

Then there exists conjugate pointpair along $\gamma$.
Proof: Suppose, on the contrary, that there cannot exist a conjugate pointpair along $\gamma$. Let the function $A(t)$, defined as

$$
A(t):=\inf _{\left[t, t_{0}\right)}\left\{\left(t-t_{0}\right)^{2} k_{E \wedge T}(t)\right\},
$$

which is continuous and increasing on $\left[t^{*}, t_{0}\right)$. Hence, there must exist such an integer $m(\geqslant 2)$ and a parameter value $t_{1} \in\left[\left(3 t^{*}+t_{0}\right) / 4, t_{0}\right)$ to this $m$ that

$$
A(t)>\left(\frac{3 m}{m-1}\right)^{2} \text {, for any } t \in\left[t_{1}, t_{0}\right) .
$$

Let $t_{2} \in\left(t_{1}, t_{0}\right)$ be defined as

$$
t_{2}:=\frac{2(m-1)}{3 m} t_{0}+\frac{(m+2)}{3 m} t_{1} .
$$

Then we have that

$$
t_{0}-t_{1}=\frac{3 m}{m-1} \frac{t_{2}-t_{1}}{2}
$$

Furthermore, by (4.3),

$$
\inf _{\left[t_{1}, t_{2}\right]}\left\{\left(t-t_{0}\right)^{2} k_{E \wedge T}(t)\right\}>\left(\frac{3 m}{m-1}\right)^{2} .
$$

Consequently,

$$
\left(t_{0}-t_{1}\right)^{2} \inf _{\left[t_{1}, t_{2}\right]}\left\{k_{E \wedge T}(t)\right\}>\left(\frac{3 m}{m-1}\right)^{2},
$$

and by the application of (4.4), we get that

$$
\inf _{\left[t_{1}, t_{2}\right]}\left\{k_{E \wedge T}(t)\right\}>\left(\frac{3 m}{m-1}\right)^{2} \frac{1}{\left(t_{0}-t_{1}\right)^{2}}=\frac{4}{\left(t_{2}-t_{1}\right)^{2}} .
$$

Then, integrating (4.6) over the interval $\left[t_{1}, t_{2}\right]$, we obtain that

$$
\int_{t_{1}}^{t_{2}} k_{E \wedge T}(t) d t \geqslant \int_{t_{1}}^{t_{2}} \inf _{\left[t_{1}, t_{2}\right]}\left\{k_{E \wedge T}(t)\right\} d t>\frac{4}{t_{2}-t_{1}} .
$$

Then, according to our choice we have that $\left[\left(3 t_{1}\right.\right.$ $\left.\left.-t_{2}\right) / 2,\left(3 t_{2}-t_{1}\right) / 2\right] \subset\left[t_{1}, t_{0}\right)$. Furthermore, the curvature function does not change its sign on $\left[\left(3 t_{1}-t_{2}\right) / 2,\left(3 t_{2}\right.\right.$ $\left.-t_{1}\right) / 2$ ]. Consequently, the conditions of Proposition 4.1.1 are satisfied, which exclude that (4.7) could be satisfied, whence the indirect condition must fail.

Corollary 4.1.3: Let $\gamma:\left(-\infty, t_{0}\right) \rightarrow M$ a future incomplete maximal causal geodesic. Suppose that there is an interval $\left[t^{*}, t_{0}\right)$ and a vector field $E$ along $\gamma$ so that the curvature function $k_{E \wedge T}(t)$ does not change sign on $\left[t^{*}, t_{0}\right)$. Then we have that 


$$
\lim _{t \rightarrow t_{0}} \inf \left\{\left(t-t_{0}\right)^{2} k_{E \wedge}(t)\right\} \leqslant 9
$$

So far we have not used any energy condition type assumption in the derivation of the above upper bound results. However, when deriving the dual lower bound results we have to impose certain restrictions on the Ricci part, $\operatorname{Ric}(T, T)$, of the tidal force tensor.

Throughout the remaining part of this paper, for a given causal geodesic $\gamma\left\{E_{i}\right\}$, we will denote one of the parallelly propagated orthonormal (resp., pseudoorthonormal) bases along $\gamma$, so that $E_{4}:=T$.

Proposition 4.1.4: Let $\gamma:[a, b] \rightarrow M$ be a maximal causal geodesic. Suppose that there exist $t_{1}, t_{2} \in(a, b)$ so that the diagonal components $R_{i 4 i 4}(t) i \in\{1,2,3\}$ (resp., $i \in\{1,2\}$ ) of the tidal force tensor for a basis $\left\{E_{i}\right\}$ do not change sign on the interval $\left[\left(3 t_{1}-t_{2}\right) / 2,\left(3 t_{2}-t_{1}\right) / 2\right]$ $C[a, b]$ and $\operatorname{Ric}(T, T)>-c /\left(t_{2}-t_{1}\right)^{2}$ along $\gamma$ for some $c \in \mathbb{R}^{+}$. Then, for any $i \in\{1,2,3\}$ (resp., $i \in\{1,2\}$ )

$$
\int_{t_{1}}^{t_{2}} R_{i 4 i 4}(t) d t>-\frac{4(n-1)+c}{t_{2}-t_{1}}
$$

where $n=3$ (resp., $n=2$ ).

Proof: Since the conditions of Proposition 4.1.1 are satisfied for the interval $\left(t_{1}, t_{2}\right) \subset[a, b]$ we have for any $i \in\{1,2,3\}$ (resp., $i \in\{1,2\}$ ) that

$$
\int_{t_{1}}^{t_{2}} R_{i 4 i 4}(t) d t<\frac{4}{t_{2}-t_{1}}
$$

Furthermore, $\operatorname{Ric}(T, T)(t) \geqslant-c /\left(t_{2}-t_{1}\right)^{2}$ along the geodesic $\gamma$. Thus we have that

$$
R_{i 4 i 4}(t) \geqslant-\sum_{j=1, j \neq i}^{n} R_{j 4 j 4}(t)-\frac{c}{\left(t_{2}-t_{1}\right)^{2}} .
$$

Then, integrating (4.11) over the interval $\left[t_{1}, t_{2}\right]$ and applying (4.10), we get that $(4.9)$ is satisfied.

Corollary 4.1.5: Let $\gamma:[a, b] \rightarrow M$ be a maximal causal geodesic. Suppose that there exist $t_{1}, t_{2} \in(a, b)$ so that the diagonal components $R_{i 4 i 4}(t) i \in\{1,2,3\}$ (resp., $i \in\{1,2\}$ ) of the tidal force tensor for a basis $\left\{E_{i}\right\}$ do not change sign on the interval $\left[\left(3 t_{1}-t_{2}\right) / 2,\left(3 t_{2}-t_{1}\right) / 2\right] \subset[a, b]$ and $\operatorname{Ric}(T, T) \geqslant-c /\left(t_{2}-t_{1}\right)^{2}$ along $\gamma$ for some $c \in \mathbb{R}^{+}$. Then, for any $i \in\{1,2,3\}$ (resp., $i \in\{1,2\}$ )

$$
\left|\int_{t_{1}}^{t_{2}} R_{i 4 ; 4}(t) d t\right|<\frac{4(n-1)+c}{t_{2}-t_{1}},
$$

where $n=3$ (resp., $n=2$ ).

In the derivation of the next statement a restriction weaker than the previous one on the Ricci part of the tidal curvature will suffice. It is said that the limes con- dition is satisfied along the future incomplete causal geodesic $\gamma:\left(-\infty, t_{0}\right) \rightarrow M$ whenever

$$
\lim _{t \rightarrow t_{0}}\left\{\left(t-t_{0}\right)^{2} \operatorname{Ric}(T, T)(t)\right\} \geqslant 0
$$

This condition is satisfied if $\operatorname{Ric}(T, T)(t)$ does not diverge too fast to $-\infty$ and, even more, if it has a lower bound along $\gamma$.

Proposition 4.1.6: Let $\gamma:\left(-\infty, t_{0}\right) \rightarrow M$ be a future incomplete maximal causal geodesic. Suppose that the limes condition is satisfied along $\gamma$ and there is an interval $\left[t^{*}, t_{0}\right)$, so that the diagonal components $R_{i 4 / 4}(t) i \in\{1,2,3\}$ (resp., $i \in\{1,2\}$ ) of the tidal force tensor for a basis $\left\{E_{i}\right\}$ do not change sign on $\left[t^{*}, t_{0}\right)$, as well as that

$$
\begin{aligned}
\lim _{t \rightarrow t_{0}} & \sup _{\left[t, t_{0}\right)}\left\{\left(t-t_{0}\right)^{2} R_{i 4 i 4}(t)\right\} \\
& \left.\quad-\inf _{\left[t, t_{0}\right)}\left\{\left(t-t_{0}\right)^{2} R_{i 4 i 4}(t)\right\}\right] \\
= & 0 .
\end{aligned}
$$

Then, for any $i \in\{1,2,3\}$ (resp., $i \in\{1,2\}$ )

$$
\lim _{t \rightarrow t_{0}}\left\{\left(t-t_{0}\right)^{2} R_{i 4 i 4}(t)\right\} \geqslant-9(n-1)
$$

where $n=3$ (resp., $n=2$ ).

Proof: Because of the above conditions, we have that

$\liminf _{t \rightarrow t_{0}}\left[t, t_{0}\right)\left\{\left(t-t_{0}\right)^{2} R_{i 4 i 4}(t)\right\}=\lim _{t \rightarrow t_{0}}\left\{\left(t-t_{0}\right)^{2} R_{i 4 i 4}(t)\right\}$,

for any value of the index $i$. Then, by the application of Corollary 4.1.3, we get that

$$
\begin{aligned}
\lim _{t \rightarrow t_{0}}\left\{\left(t-t_{0}\right)^{2} R_{i 444}(t)\right\} & \geqslant-\sum_{j=1, j \neq i \neq i}^{n} \lim _{t \rightarrow t_{0}}\left\{\left(t-t_{0}\right)^{2} R_{j 4 j 4}(t)\right\} \\
& \geqslant-9(n-1) .
\end{aligned}
$$

Then, simply as a consequence of Corollary 4.1 .3 and the above proposition, the following statement can be established.

Corollary 4.1.7: Let $\gamma:\left(-\infty, t_{0}\right) \rightarrow M$ be a future incomplete maximal causal geodesic. Suppose that the limes condition is satisfied along $\gamma$, and there is an interval $\left[t^{*}, t_{0}\right)$, so that the diagonal components $R_{i 4 i 4}(t) i \in\{1,2,3\}$ (resp., $i \in\{1,2\}$ ) of the tidal force tensor for a basis $\left\{E_{i}\right\}$ do not change the sign on $\left[t^{*}, t_{0}\right)$, as well as that 


$$
\begin{aligned}
\lim _{t \rightarrow t_{0}} & {\left[\sup _{\left[t, t_{0}\right)}\left\{\left(t-t_{0}\right)^{2} R_{i 4 i 4}(t)\right\}\right.} \\
& \left.-\inf _{\left[t, t_{0}\right)}\left\{\left(t-t_{0}\right)^{2} R_{i 4 i 4}(t)\right\}\right]=0 .
\end{aligned}
$$

Then, for any $i \in\{1,2,3\}$ (resp., $i \in\{1,2\}$ ),

$$
\left|\lim _{t \rightarrow t_{0}}\left\{\left(t-t_{0}\right)^{2} R_{i 4 i 4}(t)\right\}\right|<9(n-1)
$$

where $n=3$ (resp., $n=2$ ).

\section{B. The off-diagonal components}

As an easy application of the results of the previous section, the following two statements can be proved, in connection with the off-diagonal elements of the tidal force tensor along maximal causal geodesics.

Proposition 4.2.1: Let $\gamma:[a, b] \rightarrow M$ be a maximal casual geodesic. Suppose that there exist $t_{1}, t_{2} \in(a, b)$, so that the diagonal components $R_{i 4 i 4}(t) i \in\{1,2,3\}$ (resp., $i \in\{1,2\}$ ) of the tidal force tensor for a basis $\left\{E_{i}\right\}$ and the functions $R_{k 4 k 4}(t) \pm R_{l 4 / 4}(t)$ for some $k, l \in\{1,2,3\}$ (resp., $k, l \in\{1,2\})$ do not change the sign on the interval [ $3 t_{1}$ $\left.\left.-t_{2}\right) / 2,\left(3 t_{2}-t_{1}\right) / 2\right] \subset[a, b]$ and $\operatorname{Ric}(T, T) \geqslant-c /\left(t_{2}\right.$ $\left.-t_{1}\right)^{2}$ along $\gamma$ for some $c \in \mathbb{R}^{+}$. Then, for the indices $k, l$,

$$
\left|\int_{t_{1}}^{t_{2}} R_{k 4 / 4}(t) d t\right|<\frac{8(n-1)+2 c}{t_{2}-t_{1}}
$$

where $n=3$ (resp., $n=2$ ).

Proof: By imposing the above conditions on the components $R_{i 4 i 4}(t)_{2}$ we have that the diagonal components $R_{i 4 \pi_{i}}(t):=g\left(R\left(E_{i}, T\right) T, \widetilde{E}_{i}\right)$ of the tidal force tensor belonging to the parallelly propagated orthonormal (resp., pseudo-orthonormal) basis $\left\{\widetilde{E}_{i}\right\}$ along $\gamma$ satisfy the conditions of Corollary 4.1.5, where $\widetilde{E}_{k}:=(1 / \sqrt{2})\left(E_{k}+E_{l}\right)$, $\widetilde{E}_{l}:=(1 / \sqrt{2})\left(E_{k}-E_{l}\right)$ and $\widetilde{E}_{i}:=E_{i}$ whenever $i \neq k, l$.

Then, using the multilinearity of the curvature tensor, we have that

$$
\begin{aligned}
\int_{t_{1}}^{t_{2}} R_{\tilde{k} 4 \tilde{k} 4}(t) d t= & \frac{1}{2} \int_{t_{1}}^{t_{2}} R_{k 4 k 4}(t) d t+\frac{1}{2} \int_{t_{1}}^{t_{2}} R_{l 4 / 4}(t) d t \\
& +\int_{t_{1}}^{t_{2}} R_{k 4 / 4}(t) d t
\end{aligned}
$$

is satisfied, from which we get that

$$
\begin{aligned}
\left|\int_{t_{1}}^{t_{2}} R_{k 4 / 4}(t) d t\right| \leqslant & \left|\int_{t_{1}}^{t_{2}} R_{\tilde{k} 4 \tilde{k}_{4}}(t) d t\right|+\frac{1}{2}\left|\int_{t_{1}}^{t_{2}} R_{k 4 k 4} d t\right| \\
& +\frac{1}{2}\left|\int_{t_{1}}^{t_{2}} R_{l 4 / 4} d t\right|
\end{aligned}
$$

The statement follows from the fact that, by Corollary 4.1.5, each of the three integral terms on the righthand side of (4.16) are bounded.

Proposition 4.2.2: Let $\gamma:\left(-\infty, t_{0}\right) \rightarrow M$ be a future incomplete maximal causal geodesic. Suppose that the limes condition is satisfied along $\gamma$, and there is an interval $\left[t^{*}, t_{0}\right)$ so that the diagonal components $R_{i 4 i 4}(t) i \in\{1,2,3\}$ (resp., $i \in\{1,2\}$ ) of the tidal force tensor for a basis $\left\{E_{i}\right\}$ and the functions $R_{k 4 k 4}(t) \pm R_{14 l 4}(t)$ for some $k, l \in\{1,2,3\}$ (resp., $k, l \in\{1,2\}$ ) do not change sign on the interval $\left[t^{*}, t_{0}\right)$, as well as that

$$
\begin{aligned}
& \lim _{t \rightarrow t_{0}}\left[\sup _{\left[t, t_{0}\right)}\left\{\left(t-t_{0}\right)^{2} R_{i 4 i 4}(t)\right\}\right. \\
&\left.-\inf _{\left[t, t_{0}\right)}\left\{\left(t-t_{0}\right)^{2} R_{i 4 i 4}(t)\right\}\right]=0 .
\end{aligned}
$$

Then, for the indices $k, l$,

$$
\left|\lim _{t \rightarrow t_{0}}\left\{\left(t-t_{0}\right)^{2} R_{k 4 / 4}(t)\right\}\right| \leqslant 18(n-1),
$$

where $n=3$ (resp., $n=2$ ).

Proof: Like in the proof of the previous proposition, we have that the conditions imposed on $R_{i 4 i 4}(t)$ imply that the components $R_{i \dot{i} i 4}(t)$ satisfy the conditions of Corollary 4.1.7. Furthermore, by the multilinearity of the curvature tensor, we have that

$$
\begin{aligned}
\lim _{t \rightarrow t_{0}}\left\{\left(t-t_{0}\right)^{2} R_{\tilde{k} 4 \tilde{k} 4}(t)\right\}= & \frac{1}{2} \lim _{t \rightarrow t_{0}}\left\{\left(t-t_{0}\right)^{2} R_{k 4 k 4}(t)\right\} \\
& +\frac{1}{2} \lim _{t \rightarrow t_{0}}\left\{\left(t-t_{0}\right)^{2} R_{l 4 / 4}(t)\right\} \\
& +\lim _{t \rightarrow t_{0}}\left\{\left(t-t_{0}\right)^{2} R_{k 4 / 4}(t)\right\} .
\end{aligned}
$$

After rearrangement we get, by applying the CauchySchwartz inequality, 


$$
\begin{aligned}
& \left|\lim _{t \rightarrow t_{0}}\left\{\left(t-t_{0}\right)^{2} R_{k 4 / 4}(t)\right\}\right| \\
& \leqslant\left|\lim _{t \rightarrow t_{0}}\left\{\left(t-t_{0}\right)^{2} R_{\tilde{k} 4 \tilde{k} 4}(t)\right\}\right| \\
& +\sum_{i=k, l} \frac{1}{2}\left|\lim _{t \rightarrow t_{0}}\left\{\left(t-t_{0}\right)^{2} R_{i 4 i 4}(t)\right\}\right| \\
& \leqslant 18(n-1),
\end{aligned}
$$

where in the last line Corollary 4.1.7 was applied.

\section{CONCLUDING REMARKS}

In addition to the above-mentioned possibility of replacing the energy conditions of the classical singularity theorems by weaker ones there is another way to interpret the above results. Previously, it was shown by Tipler, ${ }^{4,6}$ Newman, ${ }^{7}$ and Szabados ${ }^{8,9}$ that there exists an upper bound to the rate of the possible divergence of the Ricci part $\operatorname{Ric}(T, T)$ or the conformal electric part of the tidal force tensor along incomplete maximal null geodesics. The statements proven in the above two sections (see, e.g., Corollaries 4.1.3 and 4.1.7 and Proposition 4.2.2) are extensions of the previous results, and show that neither of the diagonal or the off-diagonal components of the tidal force tensor that do not change sign on some final segment of $\gamma$ can blow up faster than $\left(t-t_{0}\right)^{-2}$ along maximal timelike and null geodesics. The applied limes condition is definitly weaker than the energy conditions appearing in the earlier works referred to. Furthermore, in our approach the behavior of the components can be separately examined.

It is straighforward to recast the proposed procedure for any space-time $(M, g)$ having a dimension $m \geqslant 2$. Clearly, then $n$ takes the value $n=m-1$ (resp., $n=m$
$-2)$ and the free indices $i, j, k, l$ take value from the set $\{1,2,3, \ldots, n\}$.

It is worth noting that in the derivation of our main result, see Proposition 3.3, we were strongly relying on the chosen set of functions $F[a, b]$. Since we could not find any evidence showing that our choice is the most suitable one the clearup of this problem would deserve further examination.

Note added to proof: In the proof of Lemma 3.2 we only showed that $g(t)=t / \delta$ is the only local minimum of the functional $\widetilde{I}$. However, according to Tonelli's existence theorem for Lagrange problems of the calculus variations [see, e.g., Theorem 2.20.i in Lamberto Cesari, $O p$ timization Theory and Applications (Springer-Verlag, New York, 1983)] this means that $g(t)$ is the global minimum of $\widetilde{I}$. We are grateful to Steve Harris for pointing out to us the incompleteness of the first version of the proof.

\section{ACKNOWLEDGMENT}

We would like to thank Tamás Dolinszky for reading the manuscript and suggesting a number of improvements.

${ }^{1} \mathrm{R}$. Penrose, Techniques of Differential Topology in Relativity (SIAM, Philadelphia, PA 1972); S. W. Hawking and G. R. F. Ellis, The Large Scale Structure of Spacetime (Cambridge University, Cambridge, 1973).

${ }^{2}$ S. W. Hawking and R. Penrose, Proc. R. Soc. London, Ser. A 314, 529 (1970).

${ }^{3}$ R. Geroch, Ann. Phys. 48, 526 (1968).

${ }^{4}$ F. J. Tipler, Phys. Rev. D 15, 942 (1977).

${ }^{5}$ L. B. Szabados, J. Math. Phys. 28, 142 (1987).

${ }^{6}$ F. J. Tipler, C. J. S. Clarke, and G. F. R. Ellis, in General Relativity and Gravitation, edited by A. Held (Plenum, New York, 1980).

${ }^{7}$ R. P. A. C. Newman, Gen. Rel. Grav. 16, 1177 (1984).

${ }^{8}$ L. B. Szabados, Gen. Rel. Grav, 14, 891 (1982).

${ }^{9}$ L. B. Szabados, 'Gen. Rel. Grav, 15, 187 (1983).

${ }^{10} \mathrm{P}$. E. Ehrlich and J. K. Beem, Global Lorentzian Geometry (Marcel Dekker, New York, 1981).

IIS. G. Harris, Indiana Univ. Math. J. 31, 89 (1982).

${ }^{12}$ J. Kánnár, Class. Quantum Grav. 8, L179 (1991). 